Article

\title{
Agrococcus massiliensis sp. nov., a novel bacterial species iso- lated from human healthy skin
}

\author{
Manon Boxberger ${ }^{1-2}$ : Sibylle Magnien ${ }^{1-2}$; Angéline Antezack ${ }^{1-2-3-4}$; Clara Rolland ${ }^{1-2}$; Marine Makoa ${ }^{1-}$ \\ 2 ; Bernard La Scola ${ }^{1-2}$ and Nadim Cassiri-2, \\ 1. IHU Méditerranée Infection, 19-21 Boulevard Jean Moulin, 13005 Marseille, France \\ 2. Institut de Recherche Pour le Développement (IRD), Assistance Publique-Hôpitaux de Marseille (AP-HM), \\ MEPHI, Aix-Marseille Université, 19 Boulevard Jean Moulin, 13005 Marseille, France; \\ 3. Ecole de Médecine Dentaire, Faculté des Sciences Médicales et Paramédicales, Aix-Marseille Université, \\ Boulevard Jean Moulin, 13385 Marseille, France; \\ 4. Assistance Publique-Hôpitaux de Marseille (AP-HM), Hôpital Timone, Service de Parodontologie,264, Rue \\ Saint Pierre, 13385 Marseille, France \\ *Corresponding author \\ Author details \\ Manon Boxberger manon.boxberger@hotmail.fr \\ Sibylle Magnien sibylle.magnien@gmail.com \\ Angéline Antezack angeline.a@wanadoo.fr \\ Clara Rolland rolland.clara@sfr.fr \\ Marine Makoa marine.makoa@hotmail.fr \\ Nadim Cassir cassirnadim@gmail.com \\ Bernard La Scola bernard.la-scola@univ-amu.fr
}

\begin{abstract}
Marseille-Q4369 is a strain that we isolated from human healthy skin and characterized by taxono-genomic approach. Marseille-Q4369 exhibited $99.80 \% 16$ S rRNA sequence similarity with Agrococcus pavilionensis ${ }^{T}$ the phylogenetically closest bacterium with standing in nomenclature. Furthermore, digital DNA-DNA hybridization revealed a maximum identity similarity of only $52.4 \%$ and an OrthoANI parameter provided a value of 93.63\% between the novel organism and Agrococcus pavilionensis ${ }^{T}$. Marseille-Q4369 was observed to be a yellowish-pigmented, Gram-positive, coccoïd, facultative aerobic bacterium, and belonging to the Microbacteriaceae family. The major fatty acids detected are 12-methyl-tetradecanoic acid (66\%), 14-methyl-hexadecanoic acid (24\%) followed by 13-methyl-tetradecanoic acid (5\%). The genome size of strain Marseille-Q4369 was 2,737,735-bp long with a $72,27 \% \mathrm{G}+\mathrm{C}$ content. Taken altogether, these results confirm the status of this strain as a new member of the Agrococcus genus for which the name of Agrococcus massiliensis is proposed (=CSUR-Q4369 = DSM112404).
\end{abstract}

Keywords: Bacteria; culturomics, genome, species, sp. nov., taxono-genomics 


\section{Introduction}

For several decades, with the improvement of molecular tools for bacterial identification, culture has been neglected in favor of metagenomics and 16S rRNA pyrosequencing. Since the 2010s, the culturomics approach, which is based on the diversification of culture conditions by mimicking the natural environment or unhiding the minority species through selection processes, has renewed the interest of bacterial culture as a tool to expand our knowledge on complex human microbiota [1,2]. For instance, the use of antibiotics has allowed to culture previously underestimated Gram-negative bacteria isolated from the human skin [3]. The beneficial and protective role of bacterial communities in close relationship with the skin is a challenging field of research. Recent findings represent an industrial concern through the investigation of microbial-derivate products with bioactive activities. [4]

The isolation of Agrococcus massiliensis strain Marseille-Q4369 arise as part of the culturomics project declined on the exploration of the skin microbiota. This bacterium was initially isolated from the forehead of a 59-year-old healthy woman. Here, we described this new bacterial species, using the taxono-genomics polyphasic approach, including phenotypic characterization, wall fatty acid composition and phylogenomic analyses.

\section{Results}

\subsection{Strain Marseille-Q4369 identification}

Strain Marseille-Q4369 exhibited 99.80\% 16S rRNA sequence similarity with Agrococcus pavilionensis $^{T}$, (Figure 1A) the phylogenetically closest bacterium with standing in nomenclature. Furthermore, digital DNA-DNA hybridization revealed a maximum identity similarity of only $52.4 \%$ (Figure $1 \mathrm{~B}$ and Supplementary data - Table 1).

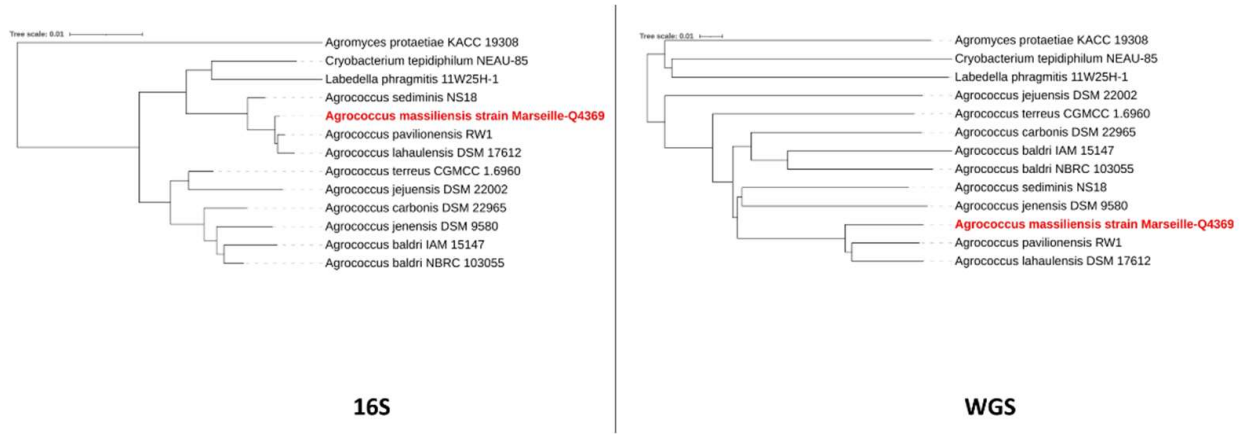

Figure 1: A) 16s rRNA-based phylogenetic tree B) Whole-genome based phylogenetic tree highlighting the position of A. massiliensis sp nov., strain Marseille-Q4369 ${ }^{\mathrm{T}}$ relative to other closely related bacterial taxa.

OrthoANI parameter provided a value of $93.63 \%$ (Figure 2) between the novel organism and Agrococcus pavilionensis ${ }^{T}$. Taken together, these results confirm the status of this strain as a new member of the Agrococcus genus for which the name Agrococcus massiliensis strain Marseille-Q4369T is proposed. 
Heatmap generated with OrthoANI values

calculated from the OAT software.

Please cite Lee et al. 2015.

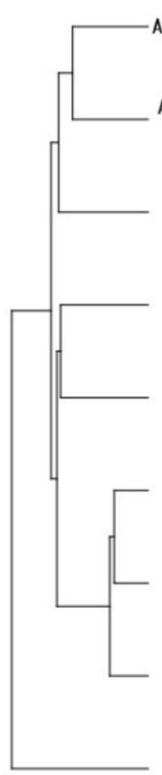

Agrococcus baldri strain IAM 15147 Agrococcus baldri strain NBRC 103
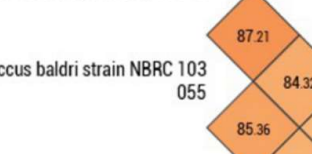

Agrococcus carbonis
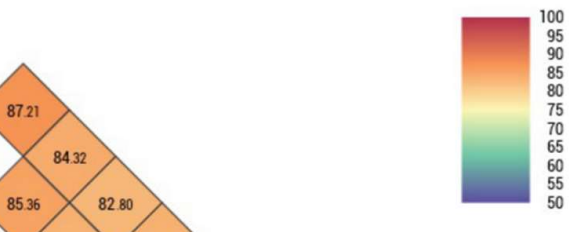

Figure 2: Heatmap generated with orthologous average nucleotide identity (OrthoANI) values calculated using the OAT software for strain Marseille-Q4369T with other closely related species validly described.

\subsection{Phenotypic characteristics of Agrococcus massiliensis strain Marseille-Q4369}

Agrococcus massiliensis strain Marseille-Q4369 was initially isolated on Columbia agar containing $5 \%$ sheep blood incubated at $31.5^{\circ} \mathrm{C}$ during 24 hours under aerobic conditions. The colonies observed are opaque yellow and not hemolytic. (Supplementary data - Figure 1B) It is non-motile (Supplementary data -Figure 1C) and did not sporulate. The cell of this strain is coccoid in shape and measures approximately $0.9 \mu \mathrm{m}$ in diameter and is gram positive. (Figure 3 and Supplementary data -Figure 1A) 


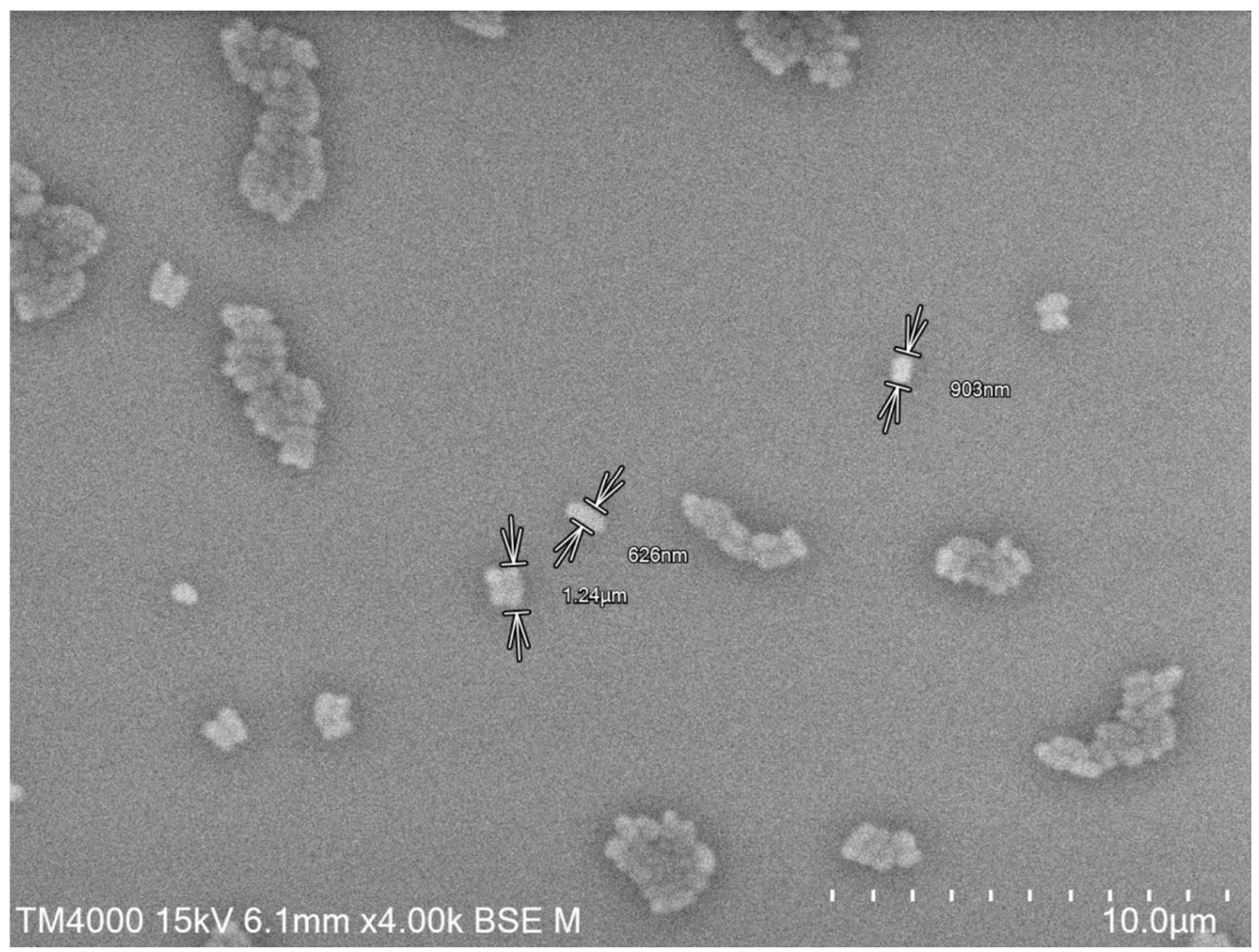

Figure 3: Scanning electron microscopy of A. massiliensis sp. nov., strain Marseille-Q4369T using a TM4000 microscope (Hitachi High-Tech, HHT, Tokyo, Japan). The scale bar represents $10 \mu \mathrm{m}$.

The bacterium is facultative aerobic, its optimal growth occurs at a $\mathrm{pH}$ between 7.5 and 8.5 , for a temperature between $31.5^{\circ} \mathrm{C}$ and $37^{\circ} \mathrm{C}$. In addition, the different concentrations of $\mathrm{NaCl}$ did not demonstrate an optimum on its growth. The fatty acids found are for the most part branched. These are 12-methyl-tetradecanoic acid (66\%), 14-methyl-hexadecanoic acid (24\%) and 13-methyl-tetradecanoic acid (5\%). A few other unsaturated and saturated fatty acids were also detected. The fatty acids found here are typical of the genus Agrococcus. (Supplementary data -Table 2)

The API galleries used for Agrococcus massiliensis are the Api ZYM, 50CH and 20NE galleries. They show positive reactions for this bacterium for the following enzymes: ferric esculin citrate, 4-nitrophenyl-\$D-galactopyranoside, D-glucose, D-maltose, malic acid, esterase (C4), leucine arylamidase, naphthol-AS-BI-phosphohydrolase and $\alpha$-glucosidase. All other reactions were negative. In addition, the reaction of catalase is positive and that of oxidase is negative.

\subsection{Genome analysis of Agrococcus massiliensis strain Marseille-Q4369}

The genome size of strain Marseille-Q4369 was 2,737,735-bp long with a 72,27 \% G+C content. The genome assembly of this strain was complete. Of the 2,744 predicted genes, 2,644 were protein-coding genes and 53 were RNAs (1 16S rRNA, 1 5S rRNAs, $123 \mathrm{~S}$ rRNAs, 47 tRNAs and 3 ncRNAs) (Figure 4) The in silico resistome of the strain Marseille-Q4369T and the search for virulence factors of this strain showed nor resistance neither virulence factor. Distribution of functional classes of predicted genes according to the clusters of orthologous groups of proteins show that the genome of Agrococcus massiliensis show a coherent structure compared to their closely related species. (Supplementary data - Figure 2) 


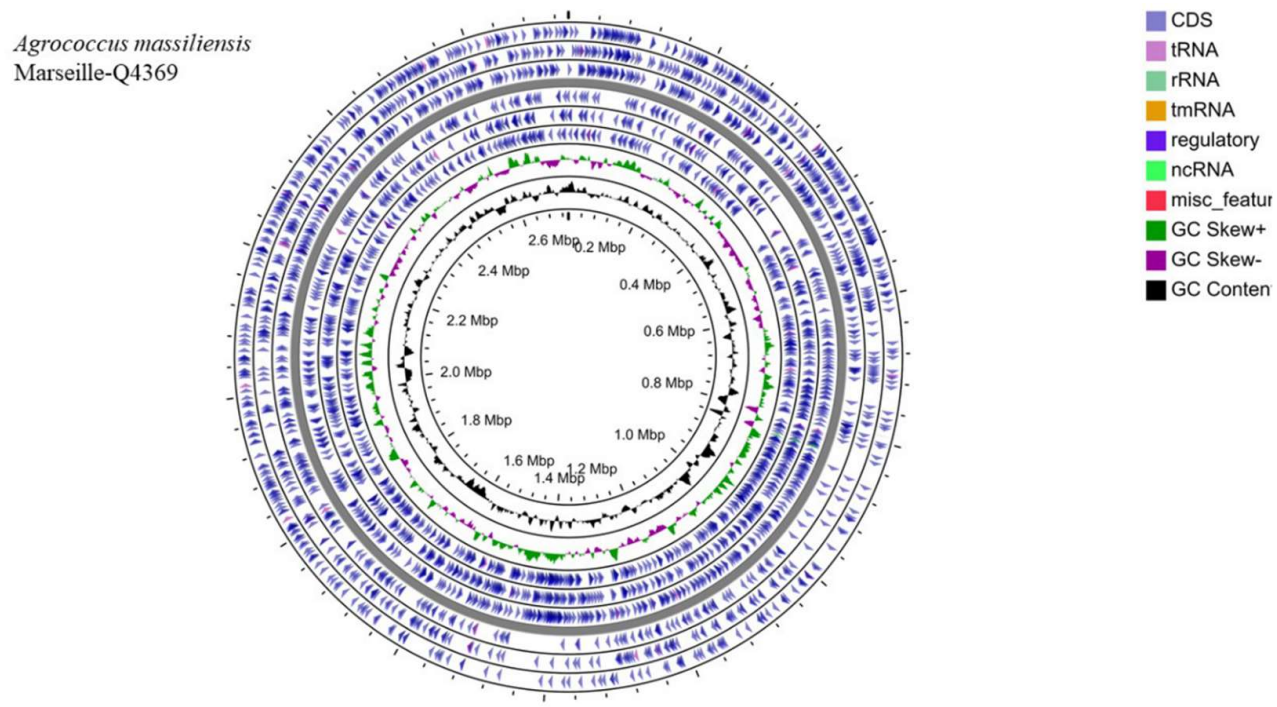

Figure 4: Graphical circular map of the genome from A. massiliensis strain MarseilleQ4369T obtained by CGView Server online tool. [27]

\section{Discussion}

Both phylogenetic and phenotypic analysis revealed several different characteristics when compared to other members of the Microbacteriaceae family, suggesting a classification as a new species of the Agrococcus genus. Agrococcus was first proposed by Groth et al. in 1996 [5]. It takes its name from the coccoid shape of its cells and from its environment, which is mostly found in soils, although it can be found in food. The genus Agrococcus comprises 11 species. Agrococcus massiliensis strain Q4369 was isolated from human healthy skin. It is the first species isolated from human. When compared to its closest bacterial species Agrococcus pavilionensisT, dDNA-DNA hybridization revealed a maximum identity similarity of only $52.4 \%$ and an OrthoANI parameter provided a value of $93.63 \%$. These gold standard methods highlight an inferior value as admitted cut-off for new bacterial species [6] that drive us to propose Marseille-Q4369 as the type strain of a new species named Agrococcus massiliensis.

Taken together, data generated by the taxonogenomic approach support that our isolate represent new species that we propose as type strains Agrococcus massiliensis.

Agrococcus massiliensis: Gr. masc. n. [ảy@ós] agros, field or soil; N.L. masc. n. coccus, coccus; from Gr. masc. n. [ко́кко丂] kokkos, grain, seed; N.L. masc. n. Agrococcus, a coccus from soil mas.si.li.en'sis. L. masc. adj. massiliensis from Massilia, the Latin name of Marseille, France, where A. massiliensis was isolated.

\section{Materials and Methods}

\subsection{Sample acquisition and strain isolation}

Samples were obtained by swabbing a $10 \mathrm{~cm}^{2}$ area of skin from the forehead and hands of volunteer healthy women. The study was validated by the ethics committee Sud-Est IV under the ID-RCB: 2019-A01508-49. Each sample mixed with the transport media was serially diluted, and $50 \mu \mathrm{L}$ of each dilution was directly seeded (DS) in Columbia agar (bioMérieux, Marcy l'Etoile, France) or homemade R2A plates , incubated under aerobic conditions at $31^{\circ} \mathrm{C}$. Plates were visualized every day until five days and subcultures were seeded in another Columbia Agar plate maintained 24 hours under aerobic conditions at $31^{\circ} \mathrm{C}$. To identify these strains, a MALDI-TOF mass spectrometry (MS) protein analysis was carried out in triplicate using a Microflex spectrometer (Bruker Daltonics, Bremen, Germany) but failed, suggesting that the generated spectra were not in the database. 
Whole Genome Sequencing (WGS) was performed as described below. Strain spectra were imported into the MALDI BioTyper software (version 3.0, Bruker, Bremen, Germany) and analyzed by standard pattern matching with default parameters. Our database (https://www.mediterranee-infection.com/acces-ressources/base-de-donnees/urms-database/) was then incremented with the spectra of these new bacterial species.

\subsection{Phenotypic tests}

Different growth temperatures $\left(20^{\circ} \mathrm{C}, 31.5^{\circ} \mathrm{C}, 37^{\circ} \mathrm{C}, 45^{\circ} \mathrm{C}\right.$ and $\left.56^{\circ} \mathrm{C}\right)$, atmosphere conditions, (anaerobic, aerobic and microaerophilic) using generator bags (CampyGEN, Oxoid, USA) and $\mathrm{pH}$ conditions $(5 ; 6.5 ; 7.5 ; 8.5)$ were tested. Biochemical properties of these strains were tested using API ZYM, API 20NE, API 20E and API 50 CH strips (bioMérieux, Marcy L'Etoile, France) according to the manufacturer's instructions. To evaluate bacterial structure, a colony was collected from agar and immersed into a $2.5 \%$ glutaraldehyde fixative solution. The suspension was vortexed, passed ten times through a 21-gauge needle to separate bacterial colonies and fixed on an uncoated glass slide by cytocentrifugation. A $1 \%$ ammonium molybdate negative stain was applied for 1 minute before gently washing the slide with $0.2 \mu \mathrm{m}$-filtered distilled water. The slide was air-dried and examined by scanning electron microscopy on a TM4000 microscope (Hitachi High-Tech, HHT, Tokyo, Japan) with a $15 \mathrm{kV}$ voltage. Motility test was performed using the semi solid TCC media as described by Tittsler et al. [7]. Sporulation was evaluated by collecting a colony from agar in $1 \mathrm{~mL}$ of Phosphate Buffer Saline (PBS) and using a thermal shock for 10 minutes at $80^{\circ} \mathrm{C}$. Cellular fatty acid methyl ester (FAME) analysis was performed by GC/MS. Two samples of each strain were prepared with approximately $110 \mathrm{mg}$ of bacterial biomass per tube harvested from several culture plates. Fatty acid methyl esters were prepared as described by Sasser (2006) [8]. GC/MS analyses were carried out as described before [9]. Briefly, fatty acid methyl esters were separated using an Elite 5-MS column and monitored by mass spectrometry (Clarus 500 - SQ 8 S, Perkin Elmer, Courtaboeuf, France). Spectral database search was performed using MS Search 2.0 operated with the Standard Reference Database 1A (NIST, Gaithersburg, USA) and the FAMEs mass spectral database (Wiley, Chichester, UK).

\subsection{Genome sequencing, annotation, and genome comparison}

Genomic DNA (gDNA) of each strain was extracted in two steps: a mechanical treatment was first performed by glass beads acid washed (G4649-500g Sigma) using a FastPrep$24^{\mathrm{TM}} 5 \mathrm{G}$ Grinder (mpBio) at maximum speed (6.5) for $90 \mathrm{~s}$. Then after 30 minutes lysozyme incubation at $37^{\circ} \mathrm{C}$, DNA was extracted on the EZ1 biorobot (Qiagen) with EZ1 DNA tissues kit. The elution volume was of $50 \mu \mathrm{L}$. gDNA was quantified by a Qubit assay with the high sensitivity kit (Life technologies, Carlsbad, CA, USA) to $0.2 \mathrm{ng} / \mu \mathrm{l}$. Genomic DNA was next sequenced on the MiSeq Technology (Illumina Inc, San Diego, CA, USA) with the paired end strategy prepared with the Nextera XT DNA sample prep kit (Illumina). To prepare the paired end library, dilution was performed to require 1ng of each genome as input to prepare the paired end library. The "tagmentation " step fragmented and tagged the DNA. Then limited cycle PCR amplification (12 cycles) completed the tag adapters and introduced dual-index barcodes. After purification on AMPure XP beads (Beckman Coulter Inc, Fullerton, CA, USA), the libraries were then normalized on specific beads according to the Nextera XT protocol (Illumina). Normalized libraries were pooled into a single library for sequencing on the MiSeq. The pooled single strand library was loaded onto the reagent cartridge and then onto the instrument along with the flow cell. To improve the quality of the assemblies, an Oxford Nanopore approach was performed on 1D genomic DNA sequencing for the MinIon device using SQK-LSK109 kit. Library 
was constructed from $1 \mu \mathrm{g}$ genomic DNA without fragmentation and end repair. Adapters were ligated to both ends of genomic DNA. After purification on AMPure XP beads (Beckman Coulter Inc, Fullerton, CA, USA), the library was quantified by a Qubit assay with the high sensitivity kit (Life technologies, Carlsbad, CA, USA). The workflow WIMP was chosen for bioinformatic analysis in live.

Genome annotation was obtained through the NCBI Prokaryotic Genome Annotation Pipeline [10]. The genome sequence data were uploaded to the Type (Strain) Genome Server (TYGS), a free bioinformatics platform available under https://tygs.dsmz.de, for a whole genome-based taxonomic analysis [11]. Determination of closest type strain genomes was done in two complementary ways: first, all user genomes were compared against all type strain genomes available in the TYGS database via the MASH algorithm, a fast approximation of intergenomic relatedness [12], and, the ten type strains with the smallest MASH distances chosen per user genome. Second, an additional set of ten closely related type strains was determined via the $16 \mathrm{~S}$ rDNA gene sequences. These were extracted from the user genomes using RNAmmer [13] and each sequence was subsequently BLASTed [14] against the 16S rDNA gene sequence of each of the currently 12983 type strains available in the TYGS database. This was used as a proxy to find the best 50 matching type strains (according to the bitscore) for each user genome and to subsequently calculate precise distances using the Genome BLAST Distance Phylogeny approach (GBDP) under the algorithm 'coverage' and distance formula d5 [15]. These distances were finally used to determine the 10 closest type strain genomes for each of the user genomes. All pairwise comparisons among the set of genomes were conducted using GBDP and accurate intergenomic distances inferred under the algorithm 'trimming' and distance formula d5. 100 distance replicates were calculated each. Digital DDH values and confidence intervals were calculated using the recommended settings of the GGDC2. Complementarily, the degree of genomic similarity of interest strains with closely related species was estimated using the OrthoANI software with default parameters [16], the closest species were determined on the DDH basis. Trees were inferred with FastME 2.1.6.1 [17] from GBDP distances calculated from $16 \mathrm{~S}$ rDNA gene sequences or whole genome sequence. The branch lengths are scaled in terms of GBDP distance formula d5. The numbers above branches are GBDP pseudo-bootstrap support values $>60 \%$ from 100 replications, with an average branch support of $84.3 \%$. The tree was rooted at the midpoint and re-generated with the iTOL Tool v5 [18]. Antibiotic resistance genes and presence of pathogenesisrelated proteins were investigated using the ABRicate tools v1.0.1 against ARG-ANNOT [19], EcOH [20], NCBI Bacterial Antimicrobial Resistance reference Gene Database [21], PlasmidFinder [22], ResFinder [23], CARD [24] and VFDB [25] using the Online Galaxy platform [26].

\section{Deposit in culture collections and Sequences Database}

Agrococcus massiliensis strain Marseille- Q4369, was deposited in CSUR collections under accession CSUR-Q4369 and DSMZ under number DSM 112404. The 16S rRNA and genome sequences are available under accession numbers MW583382 and CP070501.1 respectively.

\section{Transparency declaration}

MB is PhD granted by the collaboration between M\&L Laboratories and Aix Marseille University referenced PVM :2018-200.

\section{Funding sources}


This study was supported by the French State managed by the National Research Agency under the "Investissements d'avenir (Investments for the Future)" program under the reference ANR-10-IAHU-03 (Méditerranée Infection), by the Région ProvenceAlpes-Côte-d'Azur and the European funding FEDER PRIMI and the collaboration between M\&L Laboratories and Aix Marseille University referenced PVM :2018-200.

\section{Acknowledgements}

The authors are indebted to Ludivine BRECHARD for sequencing the genome, Nicholas ARMSTRONG and Céline CHARTIER for performing the fatty acids analyses

\section{References}

1. Wade, W.; Thompson, H.; Rybalka, A.; Vartoukian, S. Uncultured Members of the Oral Microbiome. J Calif Dent Assoc 2016, 44, 447-456.

2. Bonnet, M.; Lagier, J.C.; Raoult, D.; Khelaifia, S. Bacterial Culture through Selective and Non-Selective Conditions: The Evolution of Culture Media in Clinical Microbiology. New Microbes and New Infections 2020, 34.

3. Myles, I.A.; Reckhow, J.D.; Williams, K.W.; Sastalla, I.; Frank, K.M.; Datta, S.K. A Method for Culturing Gram-Negative Skin Microbiota. BMC Microbiol 2016, 16.

4. Boxberger, M.; Cenizo, V.; Cassir, N.; La Scola, B. Challenges in Exploring and Manipulating the Human Skin Microbiome. Microbiome 2021, 9, 125, doi:10.1186/s40168-021-01062-5.

5. Groth, I.; Schumann, P.; Weiss, N.; Martin, K.; Rainey, F.A. Agrococcus Jenensis Gen. Nov., Sp. Nov., a New Genus of Actinomycetes with Diaminobutyric Acid in the Cell Wall. Int J Syst Bacteriol 1996, 46, 234-239, doi:10.1099/00207713-46-1-234.

6. Abdallah, R.A.; Beye, M.; Diop, A.; Bakour, S.; Raoult, D.; Fournier, P.-E. The Impact of Culturomics on Taxonomy in Clinical Microbiology. Antonie van Leeuwenhoek 2017, 110, 1327-37, doi:10.1007/s10482-017-0871-1.

7. Tittsler, R.P.; Sandholzer, L.A. The Use of Semi-Solid Agar for the Detection of Bacterial Motility. J. Bacteriol. 1936, 31, 575-580.

8. Sasser, M. Bacterial Identification by Gas Chromatographic Analysis of Fatty Acids Methyl Esters (GC-FAME). MIDI 2006.

9. Dione, N.; Sankar, S.A.; Lagier, J.-C.; Khelaifia, S.; Michele, C.; Armstrong, N.; Richez, M.; Abrahão, J.; Raoult, D.; Fournier, P.E. Genome Sequence and Description of Anaerosalibacter Massiliensis Sp. Nov. New Microbes New Infect 2016, 10, 66-76, doi:10.1016/j.nmni.2016.01.002.

10. Tatusova, T.; DiCuccio, M.; Badretdin, A.; Chetvernin, V.; Nawrocki, E.P.; Zaslavsky, L.; Lomsadze, A.; Pruitt, K.D.; Borodovsky, M.; Ostell, J. NCBI Prokaryotic Genome Annotation Pipeline. Nucleic Acids Res 2016, 44, 6614-6624, doi:10.1093/nar/gkw569.

11. Meier-Kolthoff, J.P.; Göker, M. TYGS Is an Automated High-Throughput Platform for State-of-the-Art Genome-Based Taxonomy. Nat Commun 2019, 10, 2182, doi:10.1038/s41467-019-10210-3.

12. Ondov, B.D.; Treangen, T.J.; Melsted, P.; Mallonee, A.B.; Bergman, N.H.; Koren, S.; Phillippy, A.M. Mash: Fast Genome and Metagenome Distance Estimation Using MinHash. Genome Biol 2016, 17, 132, doi:10.1186/s13059-016-0997-x.

13. Lagesen, K.; Hallin, P.; Rødland, E.A.; Stærfeldt, H.-H.; Rognes, T.; Ussery, D.W. RNAmmer: Consistent and Rapid Annotation of Ribosomal RNA Genes. Nucleic Acids Research 2007, 35, 3100-3108, doi:10.1093/nar/gkm160.

14. Camacho, C.; Coulouris, G.; Avagyan, V.; Ma, N.; Papadopoulos, J.; Bealer, K.; Madden, T.L. BLAST+: Architecture and Applications. BMC Bioinformatics 2009, 10, 421, doi:10.1186/1471-2105-10-421.

15. Meier-Kolthoff, J.P.; Auch, A.F.; Klenk, H.-P.; Göker, M. Genome Sequence-Based Species Delimitation with Confidence Intervals and Improved Distance Functions. BMC Bioinformatics 2013, 14, 60, doi:10.1186/1471-2105-14-60.

16. Lee, I.; Ouk Kim, Y.; Park, S.-C.; Chun, J. OrthoANI: An Improved Algorithm and Software for Calculating Average Nucleotide Identity. International Journal of Systematic and Evolutionary Microbiology 2016, 66, 1100-1103, doi:10.1099/ijsem.0.000760. 
17. Lefort, V.; Desper, R.; Gascuel, O. FastME 2.0: A Comprehensive, Accurate, and Fast Distance-Based Phylogeny Inference Program: Table 1. Mol Biol Evol 2015, 32, 2798-2800, doi:10.1093/molbev/msv150.

18. Letunic, I.; Bork, P. Interactive Tree Of Life (ITOL) v5: An Online Tool for Phylogenetic Tree Display and Annotation. Nucleic Acids Res 2021, doi:10.1093/nar/gkab301.

19. Gupta, S.K.; Padmanabhan, B.R.; Diene, S.M.; Lopez-Rojas, R.; Kempf, M.; Landraud, L.; Rolain, J.-M. ARG-ANNOT, a New Bioinformatic Tool To Discover Antibiotic Resistance Genes in Bacterial Genomes. Antimicrob. Agents Chemother. 2014, 58, 212-220, doi:10.1128/AAC.01310-13.

20. Ingle, D.; Valcanis, M.; Kuzevski, A.; Tauschek, M.; Inouye, M.; Stinear, T.; Levine, M.M.; Robins-Browne, R.M.; Holt, K.E. EcOH: In Silico Serotyping of E. Coli from Short Read Data; Microbiology, 2015;

21. Feldgarden, M.; Brover, V.; Haft, D.H.; Prasad, A.B.; Slotta, D.J.; Tolstoy, I.; Tyson, G.H.; Zhao, S.; Hsu, C.-H.; McDermott, P.F.; et al. Validating the AMRFinder Tool and Resistance Gene Database by Using Antimicrobial Resistance Genotype-Phenotype Correlations in a Collection of Isolates. Antimicrob Agents Chemother 2019, 63, e00483-19, /aac/63/11/AAC.00483-19.atom, doi:10.1128/AAC.00483-19.

22. Carattoli, A.; Zankari, E.; García-Fernández, A.; Voldby Larsen, M.; Lund, O.; Villa, L.; Møller Aarestrup, F.; Hasman, H. In Silico Detection and Typing of Plasmids Using PlasmidFinder and Plasmid Multilocus Sequence Typing. Antimicrob. Agents Chemother. 2014, 58, 3895-3903, doi:10.1128/AAC.02412-14.

23. Bortolaia, V.; Kaas, R.S.; Ruppe, E.; Roberts, M.C.; Schwarz, S.; Cattoir, V.; Philippon, A.; Allesoe, R.L.; Rebelo, A.R.; Florensa, A.F.; et al. ResFinder 4.0 for Predictions of Phenotypes from Genotypes. Journal of Antimicrobial Chemotherapy 2020, dkaa345, doi:10.1093/jac/dkaa345.

24. McArthur, A.G.; Waglechner, N.; Nizam, F.; Yan, A.; Azad, M.A.; Baylay, A.J.; Bhullar, K.; Canova, M.J.; De Pascale, G.; Ejim, L.; et al. The Comprehensive Antibiotic Resistance Database. Antimicrob. Agents Chemother. 2013, 57, 3348-3357, doi:10.1128/AAC.00419-13.

25. Chen, L. VFDB: A Reference Database for Bacterial Virulence Factors. Nucleic Acids Research 2004, 33, D325-D328, doi:10.1093/nar/gki008.

26. Afgan, E.; Baker, D.; van den Beek, M.; Blankenberg, D.; Bouvier, D.; Čech, M.; Chilton, J.; Clements, D.; Coraor, N.; Eberhard, C.; et al. The Galaxy Platform for Accessible, Reproducible and Collaborative Biomedical Analyses: 2016 Update. Nucleic Acids Res 2016, 44, W3-W10, doi:10.1093/nar/gkw343.

27. Grant, J.R.; Stothard, P. The CGView Server: A Comparative Genomics Tool for Circular Genomes. Nucleic Acids Research 2008, 36, W181-W184, doi:10.1093/nar/gkn179. 